\title{
Los micromachismos en los adolescentes. Su asociación con las relaciones de pareja y el modelo de maternidad y paternidad
}

\author{
Everyday sexism in teenagers. The association with relationships and the \\ maternity and paternity model
}

\section{Os micromachismos nos adolescentes. Sua associação com as relações de casal e o modelo de maternidade e paternidade}

\author{
Alba García Campaña1, Marta Hidalgo Lacalle², María del Carmen López \\ León ${ }^{3}$, María del Rocío Román Almendros ${ }^{4}$ \\ ${ }^{1}$ Enfermera especialista en Obstetricia y Ginecología. Licenciada en Antropología Social y \\ Cultural. Hospital General Universitario Gregorio Marañón. \\ ${ }^{2}$ Enfermera especialista en Obstetricia y Ginecología. Hospital General Universitario Gregorio \\ Marañón. \\ ${ }^{3}$ Enfermera especialista en Obstetricia y Ginecología. Hospital General Universitario Gregorio \\ Marañón. \\ ${ }^{4}$ Enfermera especialista en Obstetricia y Ginecología. Hospital General Universitario Gregorio \\ Marañón. \\ García Campaña, A; Hidalgo Lacalle, M; López León, MC; Román Almendros, MR.
}

Cómo citar este artículo en edición digital: García Campaña, A., Hidalgo Lacalle, M., López León, Mạ C., \&

Román Almendros, Mạ.R. (2018). Los micromachismos en los adolescentes. Su asociación con las relaciones de pareja y el modelo de maternidad y paternidad. Cultura de los Cuidados (Edición digital), 22(51). Recuperado de <http://dx.doi.org/10.14198/cuid.2018.51.16> Correspondencia: Alba García Campaña. C/ Vista Alegre № 3B, 2A 28019 Madrid

Correo electrónico: albagarcia.due@gmail.com Recibido: 18/11/2017; Aceptado: 13/04/2018.

\begin{abstract}
Female identity is still represented by an emotional dependency, empathy and taking care of others, meanwhile the male one is still associated to self-sufficiency, rationality, selfcontrol, strength, power and competitiveness. Everyday sexim are male domination practices on their daily life, precisely consolidated on the social/cultural subtleness, almost imperceptible, practiced even by "the less of the world's male chauvinist”. In recent studies about Spanish youth, it is noticed the persistence of traditional
\end{abstract}

gender roles. Objective. Recognize social representations of the everyday sexim inside the couple model and the concept teenagers have about paternity/maternity in the Center district of Madrid. Methodology. Qualitative study where two different discussion groups were created, one with boys and the other one with girls. Conclusion. The results of such study show a teenager population knowledgeable of the male chauvinist matters, and become aware to make a change towards a more equal society. It is shown the importance the education has in equality at early ages to achieve a change in the society. It 
is important to carry on exploring the youngers point of view regarding these matters, to get to know up to what point they are truly changing their perspective.

Keywords: Qualitative analysis, everyday sexism, adolescent, family relations, paternity.

\section{RESUMEN}

La identidad femenina continúa representada por la dependencia emocional, la comprensión y el cuidado de los otros, mientras que la masculina sigue asociada a la autosuficiencia, la racionalidad, el control emocional, la fuerza, el dominio y la competitividad. Los micromachismos son prácticas de dominación masculina en la vida cotidiana, precisamente cimentadas en la sutileza social/cultural, casi imperceptible, que ejerce hasta el "hombre menos machista del mundo”. Informes recientes sobre la juventud en España muestran la persistencia de los roles de género tradicionales. Objetivo. Conocer las representaciones sociales de los micromachismos en el modelo de pareja y el concepto de maternidad/paternidad que tienen los adolescentes del distrito Centro de Madrid. Metodología. Se trata de un estudio cualitativo en el que se realizaron dos grupos de discusión, uno con chicos y uno con chicas. Conclusiones. Los resultados muestran una población adolescente conocedora sobre temas de machismo y concienciada a producir un cambio hacia una sociedad más igualitaria. Se observa la importancia que tiene la educación en igualdad a edades tempranas para conseguir un cambio en la sociedad. Es importante continuar explorando los puntos de vista de los jóvenes sobre estos temas para conocer hasta qué punto está cambiando su perspectiva.

$\begin{aligned} & \text { Palabras clave: Análisis cualitativo, } \\ & \text { micromachismos, adolescente, } \\ & \text { familiares, paternidad. }\end{aligned}$

\section{RESUMO}

A identidade feminina continua representada pela dependência emocional, pela compreensão e pelo cuidado dos outros, enquanto que a masculina permanece associada à autosuficiência, à racionalidade, ao controlo emocional, à força, ao domínio e à competitividade. Os micromachismos são práticas de domínio masculino na vida quotidiana, precisamente cimentadas na subtileza social/cultural, quase impercetível, que exerce até o "homem menos machista do mundo”. Relatórios recentes sobre a juventude em Espanha mostram a persistência dos papéis de género tradicionais. Objetivo. Conhecer as representações sociais dos micromachismos no modelo de casal e o conceito de maternidade/paternidade que têm os adolescentes do distrito Centro de Madrid. Metodologia. Trata-se de um estudo qualitativo no qual se realizaram dois grupos de discussão, um com rapazes e outro com raparigas. Conclusões. Os resultados mostram uma população adolescente conhecedora de temas sobre machismo e consciencializada para produzir uma mudança no sentido de uma sociedade mais igualitária. Observa-se a importância que tem a educação em igualdade em idades precoces para conseguir uma mudança na sociedade. É importante continuar a explorar os pontos de vista dos jovens sobre estes temas para conhecer até que ponto está a mudar a sua perspetiva.

Palavras-chave: Análise qualitativa, micromachismos, adolescente, relações familiares, paternidade.

\section{INTRODUCCIÓN}

\section{Sobre el género y la masculinidad}

El sujeto se construye a partir de la interacción de tres esferas: biológica, las diferencias anatomofisiológicas que determinan la adscripción a un sexo biológico (macho, hembra o intersexos); social, los roles de mujeres y hombres que aprendemos en la socialización; y psíquica, donde se construyen las posiciones subjetivas femeninas y masculinas a partir de los modelos ideales de la sociedad (Velasco Arias, 2009). El género es una construcción cultural basada en las diferencias objetivas entre sexos. A partir de estas diferencias cada cultura determina las categorías de sexo y de género. «La identidad femenina continúa representada por la dependencia emocional, la comprensión y el cuidado de los otros [...]. La identidad masculina está asociada a la autosuficiencia, la 
racionalidad, el control emocional, la fuerza, el dominio y la competitividad. Su socialización [...] está orientada a que repriman las emociones y recurran a la violencia» (Yago Simón, 2011).

En el artículo de Fernando Hernández et ál. los chicos participantes hablan de la debilidad de las mujeres, algo que está muy naturalizado en los medios de comunicación y que conduce a la superioridad del hombre respecto a la mujer. Algún chico refiere sentirse incómodo si alguna chica es mejor que él en algún deporte. El deporte es uno de los lugares en los que se construye la masculinidad hegemónica (Hernández et al, 2007). El hecho de que los hombres tengan el poder que va ligado a la masculinidad también tiene un coste, aunque no sea comparable a la opresión que sufren las mujeres. «La experiencia contradictoria del poder entre los hombres». Para la mayoría de los hombres es imposible cumplir los requisitos de los ideales dominantes de la masculinidad, pero estos mantienen una poderosa e inconsciente presencia. El patriarcado no solo mantiene la jerarquía de poder entre hombres y mujeres, sino también entre distintos grupos de hombres y masculinidades. Así los hombres suprimen toda una gama de emociones asociadas a la feminidad (Kaufman, 1999).

\section{Sobre los micromachismos}

Existen una serie de comportamientos masculinos cuyo objetivo es la exclusión de la mujer del territorio del poder. Un ejemplo serían los directivos varones que se reúnen de forma informal fuera del trabajo para crear influencias, excluyendo a las mujeres que puedan no acudir por las cargas familiares. Este tipo de comportamientos masculinos ha recibido distintos nombres. Luis Bonino acuñó el término micromachismos en 1990 (Bonino, 2008). Los micromachismos son «prácticas de dominación masculina en la vida cotidiana, precisamente cimentadas en la sutileza social/cultural, casi imperceptible, lo que está en los límites de la evidencia, [...] que ejerce hasta el "hombre menos machista del mundo”, cuyo estilo de vida de primera mano pudiera parecer muy lejano del modelo masculino del siglo pasado. Y no es que represente en sí mismo una amenaza. Su bagaje, la conciencia (o subconciencia) de proveer, proteger, ser "la cabeza”, lo llevan a aplicar los micromachismos en un contexto que parece benévolo porque es tan cotidiano que parece bueno o normal» (Ramírez García, 2013). Los micromachismos se clasifican en cuatro categorías: utilitarios, tratan de forzar la disponibilidad femenina aprovechándose de diversos aspectos «domésticos y cuidadores» del comportamiento femenino tradicional; encubiertos, intentan ocultar su objetivo abusando de la confianza y credibilidad femeninas; de crisis, intentan forzar la permanencia en el statu quo desigualitario cuando este se desequilibra; y coercitivos, que usan la fuerza psicológica o moral masculina (Bonino, 2008).

La violencia de género se manifiesta como el símbolo más brutal de la desigualdad existente en nuestra sociedad. La violencia de género tiene su base en la transmisión de los valores de dominación masculina sobre la mujer, y por este motivo hay que tratar primero las actitudes, creencias, prejuicios y mitos que legitiman la desigualdad, la subordinación o la inexistencia simbólica de las mujeres (Bonino, 2008). En el estudio de Bosch Fiol et ál. se evaluaron las actitudes y la aceptación social de los micromachismos y la relación socialmente percibida entre estos y el mantenimiento de la violencia contra las mujeres en la pareja. Se elaboró un cuestionario a partir de la clasificación de Luis Bonino, y se entrevistó a hombres y mujeres de las distintas comunidades autónomas. Algunas de las conclusiones obtenidas fueron: los micromachismos de relegación de las mujeres al rol tradicional son aceptables para más de un $40 \%$ de las personas, mientras que los que implican generación de inseguridad y temor son aceptables para más del 20\%; los varones muestran niveles de acuerdo significativamente superiores a las mujeres; y a mayor edad, mayor es la aceptación (Bosch Fiol et al, 2007).

Muñiz Tinajero, en su trabajo final del máster Mujeres y Salud, valoró la satisfacción de 29 parejas en función de la existencia de micromachismos y de la sensibilidad en perspectiva de género. Obtuvo los siguientes resultados: A mayor nivel de micromachismos, menor es la satisfacción en la pareja; y a mayor sensibilidad en perspectiva de género, menor es la satisfacción en la pareja, y viceversa. 
Estos datos son incluso más significativos entre los hombres que entre las mujeres. El autor propone 3 teorías para explicarlo: respuesta socialmente aceptada de los hombres, aceptación de los micromachismos por parte de las mujeres a pesar de identificarlos, o generación de una mayor conciencia por parte de los hombres, teniendo en cuenta q la mayoría tenían formación universitaria (Muñiz Tinajero, 2012).

\section{Sobre los jóvenes, la igualdad y sus concepciones sobre la pareja y la familia}

En el Informe de la Juventud en España en 2012 se recogen los siguientes datos: Un 48,2\% de las mujeres consideran la maternidad como un obstáculo para su profesión, mientras que solo un 24,1\% de los hombres lo considera; España es uno de los países europeos donde mayor es la diferencia de tiempo dedicado a las labores del hogar entre hombres y mujeres; un $69,4 \%$ de la sociedad considera la familia ideal aquella en la que el hombre y la mujer trabajan fuera de casa y se reparten el cuidado de los hijos y las tareas domésticas (porcentaje mayor entre las mujeres y más aún entre los jóvenes); y en contraposición, el 35,7\% de los jóvenes de entre 18 y 30 años (porcentaje incluso mayor entre las mujeres) piensa que debe ser la mujer la que reduzca su jornada laboral para ocuparse de los hijos (Moreno Mínguez et al, 2013).

En el estudio «La evolución de la adolescencia española sobre la igualdad y la prevención de la violencia de género», realizado a estudiantes de entre 12 y 24 años, se obtuvieron los siguientes resultados: el $11,67 \%$ de los chicos y el $3 \%$ de las chicas están de acuerdo con que los chicos salgan con muchas chicas pero no al revés; el $9,25 \%$ de los chicos y el $6,54 \%$ de las chicas dicen que el hombre que parece agresivo es más atractivo; y el 9,12\% de los chicos y el 5,54\% de las chicas creen que si una mujer es maltratada y no deja a su pareja es porque no le disgusta del todo (Delegación del Gobierno para la Violencia de Género, 2013).

En el estudio «Juventud Universitaria ante la Igualdad y la Violencia de Género» realizado por el Ministerio de Sanidad, Servicios Sociales e Igualdad, se vio que: El 5,73\% de los chicos y el 1,88\% de las chicas están de acuerdo con que los chicos salgan con muchas chicas pero no al revés; el 20,01\% de los chicos y el 9,42\% de las chicas están de acuerdo con que el hombre que parece agresivo es más atractivo; y el 5,34\% de los chicos y el 2,11\% de las chicas están de acuerdo con que si una mujer maltratada no abandona a su pareja es porque no le disgusta del todo (Delegación del Gobierno para la Violencia de Género, 2012).También observaron que: El $52,70 \%$ de los chicos y el $40,05 \%$ de las chicas dicen que las mujeres deben ser queridas y protegidas por los hombres. Y el $14,37 \%$ de los chicos y el 4,64\% consideran que los hombres deben dar seguridad económica a las mujeres (Delegación del Gobierno para la Violencia de Género, 2012).

\section{Concepciones de maternidad/paternidad en los adolescentes}

\section{- $\quad$ Concepciones sobre la maternidad}

«La maternidad no es sólo un producto resultante del ejercicio de la sexualidad, una consecuencia biológica de practicar sexo. La maternidad está asociada culturalmente a la feminidad y al ser mujer, a la naturaleza» (Federación de Planificación Familiar Estatal, 2012).

«Si hay algún determinante de género por excelencia para las mujeres, éste es el mandato de ejercer el rol materno, como finalidad o proyecto de vida, como dador de reconocimiento social y como proveedor de identidad propia [...] "se es mujer cuando se es madre" " (Yago Simón, 2011).

\section{- Concepciones sobre la paternidad}

«Los hombres han incursionado de manera más afectiva en la paternidad, sin embargo continúan conservando algo de distancia, lo que es propio de la caracterización de su género [...].En el caso de los varones se espera que el papel en la familia sea principalmente el de proveedor económico [...], mientras que encargarse de la protección y el mantenimiento de los hijos es algo asignado a las mujeres» (Anabalón et al, 2011).

«Si bien es cierto que los hombres han comenzado a mostrar cambios en la percepción del rol paterno, con mayor deseo de compartir el cuidado de los hijos [...], la condición oficial del 
padre proveedor no tiene discusión y adquiere una importancia fundamental. El padre puede estar ausente afectivamente, pero "si es un buen proveedor, es un buen padre» (Romero von Buchwald, 2012).

\section{OBJETIVOS}

\section{Objetivo general}

Conocer las representaciones sociales de los micromachismos en el modelo de pareja y el concepto de maternidad/paternidad que tienen los adolescentes del distrito Centro de Madrid.

\section{Objetivos específicos}

- Conocer las representaciones sociales de los micromachismos por parte de los adolescentes.

- Distinguir cuál es el modelo de pareja ideal para los adolescentes.

- Conocer cuáles son las concepciones que tienen los adolescentes sobre los roles de maternidad y paternidad.

- Examinar la aceptación y papel de los micromachismos en el seno de las concepciones más tradicionales de maternidad y paternidad.

\section{METODOLOGÍA}

\section{Diseño}

Se trata de un estudio con abordaje cualitativo. Se realizó con adolescentes del distrito centro de Madrid, con la colaboración del Centro Madrid Salud Joven del distrito centro de Madrid y el Instituto de educación secundaria Santa Teresa del distrito centro de Madrid, que cedió una de sus aulas para la realización de los grupos de discusión.

\section{Población de estudio}

La población de estudio fueron chicos y chicas de clase media que vivían y estudiaban en el distrito centro del ayuntamiento de Madrid. Para seleccionar la muestra se empleó un muestreo no probabilístico en el que los sujetos fueron elegidos según propósito o conveniencia según los siguientes criterios:
- Criterios de inclusión: Tener entre 16 y 20 años de edad, ser español de nacimiento y saber hablar castellano.

- Criterios de exclusión: No aceptar participar en el estudio y/o no acudir a la cita.

\section{Metodología}

Se eligió como técnica de recogida de datos el grupo de discusión por ser el método idóneo para captar las imágenes sociales, las representaciones culturales y los valores dominantes en un conjunto de individuos. Se realizaron dos grupos de discusión, uno compuesto por 7 chicas y el otro compuesto por 7 chicos. Durante la realización de los grupos de discusión, se procedió a la grabación de audio de las intervenciones de los participantes, con el fin de poder transcribirlas y analizarlas.

\section{Análisis de datos}

Tras la obtención de los datos, se transcribieron y ordenaron, para después poder codificar la información, concentrando las ideas y conceptos similares que se obtuvieron de las conversaciones llevadas a cabo en los grupos de discusión. Posteriormente se redactó el informe de resultados contestando a los objetivos planteados.

\section{Consideraciones éticas}

Este estudio fue presentado al Comité de Ética de Investigación Clínica del Hospital General Universitario Gregorio Marañón y fue aprobado para su realización. El tratamiento, la comunicación y la cesión de los datos de carácter personal de todos los sujetos participantes, se ajustó a lo dispuesto en la Ley Orgánica 15/1999, de 13 de diciembre de protección de datos de carácter personal.

\section{RESULTADOS Y DISCUSIÓN}

Se ha contado para este estudio con la participación de un total de 14 sujetos adolescentes, 7 chicas y 7 chicos. En el momento del estudio, las chicas tenían entre 16 y 19 años, y las chicos entre 17 y 18 años. Respecto a su nivel de estudios, 3 de las chicas participantes eran estudiantes de ESO, mientras 
que las 4 restantes eran estudiantes de bachillerato, y todos los chicos estudiaban bachillerato.

Tras analizar los discursos de los distintos sujetos en los grupos de discusión, se encontraron los siguientes resultados para cada uno de los objetivos:

\section{Conocer las representaciones sociales de los micromachismos por parte de los adolescentes.}

\section{$>$ Micromachismos}

El grupo de las chicas se posicionó mayoritariamente en contra de los micromachismos, señalando por ejemplo la importancia de que las tareas domésticas no sean responsabilidad de las mujeres y que los hombres ayuden, sino que sean algo repartido.

Sin embargo, una de las chicas participantes, se mostró en desacuerdo, señalando que muchas mujeres desatienden sus obligaciones en las tareas domésticas cargando a su pareja que trabaja. Ante estas afirmaciones, la opinión mayoritaria de chicas fue contraria, señalando además que no se trata de algo generalizado, sino que la generalidad es que las mujeres siguen siendo más responsables de las tareas del hogar.

La opinión mayoritaria en el grupo de los chicos, al igual que en el de las chicas, fue a favor de un reparto equitativo de las tareas domésticas.

Se observa que nuestros sujetos se ajustan en general al modelo igualitario señalado por Velasco Arias como el horizonte hacia el que tender por ser protector de género.

El estudio de Bosch et ál. para evaluar la aceptación social de los micromachismos, concluyó que los varones aceptaban más los micromachismos que las mujeres y que a mayor edad más micromachistas eran. Esto se cumple en nuestro estudio dado que nuestros sujetos son adolescentes. Sin embargo, no se ve la diferencia esperada entre el grupo de chicos y el de chicas. Incluso en algunos aspectos, ellos aceptaron en menor grado que ellas determinadas cosas. Esto mismo le ocurrió a Muñiz Tinajero en su trabajo final del máster. A las hipótesis que él propone para explicarlo, podríamos añadir una cuarta opción dadas las peculiaridades de este estudio: dos de los participantes en el grupo de discusión de los chicos manifestaron ser homosexuales y es posible que a lo largo de su vida hayan sufrido las consecuencias de la construcción de género que diferencia entre masculinidad hegemónica y feminidad.

Sobre los micromachismos, los chicos se centraron en si los micromachismos son machismo o no. Entre todos, reflexionaron sobre varias cosas de su vida cotidiana que consideraban micromachismos, y todo estuvieron de acuerdo en considerarlo machismo.

"El hacer eso, aunque lo hagas inconscientemente, es algo machista. Que seas machista no quiere decir que digas "soy un machista” [...] Supongo que habrá gente que sí, pero el $90 \%$ de los hombres no les gusta ser machistas. Sin embargo, estamos viendo cómo es la sociedad. Y entonces, sí que somos machistas aunque no queramos serlo. Inconscientemente” (Grupo chicos)

Sus opiniones coinciden con la definición que da el propio Luis Bonino. Los tipos de micromachismos más identificados fueron los utilitarios, los relacionados con las tareas domésticas y la función cuidadora de la mujer. Los otros tipos no fueron identificados.

\section{$>$ Masculinidades y feminidades}

En ambos grupos se reflexionó bastante sobre las diferencias entre hombres y mujeres, y a que se deben éstas. El discurso hegemónico en ambos grupos fue cómo a través de la televisión, de la publicidad o de los juguetes divididos por género, te van introduciendo micromachismos que la sociedad va adoptando como algo normal.

"Y en las páginas de juguetes y todo eso, la parte rosa es de las chicas y la parte azul es de los chicos. $Y$ en la de las chicas te vienen cocinitas, te viene no sé qué, maquillaje... Y a los chicos, coches, motos, cosas de construcción [...] y es un micromachismo..." (Grupo chicas)

Sus opiniones coinciden en gran medida con las teorías expuestas por Yago Simón. 
A este respecto, uno de los chicos contó que cuando era pequeño, no le habían dejado anotarse a clases de ballet, mientras su hermana sí pudo anotarse a clases de danza. Otro expuso que cuando era pequeño a él le gustaban las muñecas de su hermana, pero todo el mundo le regalaba cosas de deporte. Esto coincide con lo que se habla en el artículo de Hernández et ál. sobre la importancia del deporte en la construcción de una masculinidad hegemónica.

Michael Kaufman habla en su artículo de la masculinidad hegemónica, no solo en contraposición a la feminidad, sino también en contraposición a otros tipos de masculinidades, como puede ser el caso de la homosexualidad. El hecho de que dos de nuestros sujetos fueran homosexuales, puede que les haya influido a la hora de tener una perspectiva más crítica sobre el tema.

Hablando sobre el pequeño porcentaje de las mujeres en puestos de responsabilidad en empresas, uno de los chicos manifestó que los hombres son más educados en el poder que las mujeres. Entonces, otro de los chicos expuso que esa también es la razón por la que las mujeres son más sentimentales, porque les enseñan a eso.

"Yo lo relaciono con la mítica de la sensibilidad y demás para las mujeres. La agresividad, la fuerza física, como profesores de educación física, todo... O sea, todas mujeres, menos los de educación física que son hombres. Y bomberos, policías, militares, suelen ser hombres por eso...” (Grupo chicos)

El discurso principal de la mayoría fue que si hubieran recibido otra educación, seguramente tendrían otros gustos distintos a los actuales.

\section{Distinguir cuál es el modelo de pareja ideal para los adolescentes.}

$>$ Diferencias de roles según género en la pareja

La posición mayoritaria en ambos grupos fue que la pareja ideal debía ser igualitaria, descartando la idea de que el hombre trabaje de forma remunerada fuera de casa mientras la mujer se queda en casa cuidando de la casa y de los niños.
Esta idea fue especialmente rechazada por el grupo de las chicas, que no concebían la idea de no trabajar fuera de casa de forma remunerada en el futuro. Alguna de ellas recalcaba la importancia de la independencia económica ante una futura separación.

Esto coincide con los resultados del Informe de la Juventud en España realizado en 2012, en el que dicen que esto se piensa más entre los jóvenes.

Aunque no fue una opinión unánime, si se pudo ver en varias de las participantes del grupo una cierta infravaloración del trabajo doméstico, equiparándolo a estar «sin hacer nada».

En el siguiente verbatim se pueden observar los dos puntos anteriores:

"Pero por ejemplo, si yo decido no trabajar, y estoy con una pareja y él es el que me mantiene, ¿qué pasa cuando yo me separe? No he trabajado en mi vida, no tengo no sé qué,... Pues si es algo que has decidido tú, tienes que cargar con tus consecuencias. Si es algo que has querido tú claro, no lo sé. Si hay hechos que hayan forzado esa situación, pues ya sería distinto. Pero si tú estás seguido tocándote las narices, pues luego carga las consecuencias de tocarte las narices" (Grupo chicas)

$>$ Mito del amor romántico

La posición mayoritaria a este respecto fue la misma en los dos grupos, en contra de la idea de la media naranja y del poder del amor que todo lo supera. Pero no fue unánime. Ante la siguiente afirmación de una de las chicas sobre el amor romántico: “...en lo más íntimo de la pareja, pues yo me imagino una relación donde ambos son detallistas con el otro, pero que la mujer es la princesa y el hombre el que la mima...”, la mayoría del grupo de posicionó en contra, afirmando que nos condicionan a creer en la media naranja, pero que no es necesario vivir en pareja para ser feliz y sentirse realizado:

“...desde siempre, es como que ya desde que naces te han enseñado a que como eres la media naranja de uno, tienes que buscar a tu otra media naranja para complementarte y no sé qué. Pero es que eso no es así, tú puedes estar... vivir 
sola la vida sin tener que estar en una relación o tener hijos o casarte, o cualquier cosa..." (Grupo chicas)

Aunque en nuestro grupo solo una persona habló de la importancia del amor romántico, se ve que el mito del amor romántico sigue teniendo importancia. Coincide con los resultados del estudio de «Juventud Universitaria ante la Igualdad y la Violencia de Género».

> Relaciones controladoras y violencia machista

La posición mayoritaria de las chicas sobre las relaciones controladoras fue de desagrado. Analizaron las razones por las que una mujer aguanta una situación así, nombrando causas como el amor, el miedo, la soledad,... Una de las chicas se mostró en disconformidad, exponiendo la parte de culpabilidad que tiene la mujer al consentirlo:

"Yo creo que eso es porque las parejas mujeres con otros hombres lo permiten. Si no, no tendría porque ser así” (Grupo chicas)

Esto coincide con los resultados del estudio «La evolución de la adolescencia española sobre la igualdad y la prevención de la violencia de género», y del estudio «Juventud Universitaria ante la Igualdad y la Violencia de Género».

Conocer cuáles son las concepciones que tienen los adolescentes sobre los roles de maternidad y paternidad, y examinar la aceptación y papel de los micromachismos en el seno de las concepciones más tradicionales de maternidad y paternidad.

Ninguno de los sujetos de los dos grupos asoció la paternidad con ser proveedor económico ni la maternidad con cuidado específicamente. Ambos recalcaban esas funciones como importantes tanto para la madre como para el padre de forma indistinta.

“...por ejemplo, el marido y la mujer trabajan, uno trae más dinero que otro, entonces yo creo que uno debería aportar más dinero que el otro. $Y$ respecto de las tareas, pues por ejemplo, el ir a comprar pañales o dar el biberón y todo eso, pues se lo deberían repartir. No tiene que ir solo la madre" (Grupo chicos)

Observamos que entre las chicas de nuestro estudio no había una relación directa entre su identidad como mujeres y la necesidad de ser madres, al contrario que en el estudio de la Federación de Planificación Familiar Estatal del proyecto europeo SAFE II.

Yago Simón recalca también este aspecto de la maternidad como finalidad y proyecto de vida y como proveedor de identidad propia. No se observa este pensamiento en las chicas participantes de nuestro estudio.

Los chicos reclaman una baja de paternidad más larga, ya que la actual impide una mayor implicación emocional y en el cuidado del padre con sus hijos. Argumentan que la sociedad atribuye la función de cuidar a las mujeres:

"A mí lo que me parece muy curioso es que tú vas a un baño, y está el baño de mujeres y está el baño de hombres. Supongamos que vas con un niño. Si eres hombre, no puedes entrar al baño de mujeres a eso de cambiar pañales porque está en el de mujeres y en el de mujeres no puedes entrar. Pero en el de hombres no hay" (Grupo chicos)

Rechazan que esto sea así, recalcando que ellos consideran que las funciones de maternidad y paternidad deberían ser igualitarias.

En el estudio de Anabalón et ál. se habla de una nueva paternidad caracterizada por una concepción donde los adolescentes presentan mayor compromiso emocional y afectivo, más presencia en la crianza y en el desarrollo de los hijos, pero manteniendo algunos aspectos del modelo antiguo de padre como el de sostenedor económico. Esto mismo dice Romero von Buchwald, en su tesis de grado. Encontramos entre los chicos de nuestro estudio este deseo de mayor participación en el cuidado de los hijos, pero no la importancia del papel de proveedor económico.

\section{CONCLUSIONES}

Los resultados muestran una población adolescente conocedora sobre temas de 
machismo y concienciada a producir un cambio hacia una sociedad más igualitaria.

Varios de los sujetos manifestaron haber recibido algún taller o charla sobre el machismo, que les permite tener más conocimientos y pensamiento crítico al respecto.

Todo esto lleva a que sus ideas sobre las relaciones de pareja, sobre la familia ideal o sobre las concepciones sobre la maternidad y la paternidad estén más próximas a un modelo igualitario, el protector de género. Se demuestra la importancia que tiene la educación en igualdad a edades tempranas para conseguir un cambio en la sociedad.

\section{BIBLIOGRAFÍA}

Anabalón, C., Cares, F., Cortés, R., \& Zamora, M. (2011). Construcción de la propia paternidad en adolescentes varones pertenecientes a liceos municipales de la comuna de La Cisterna. Revista de Psicología, 20(1), 53-72.

Bonino, L. (2008). Hombres y violencia de género. Más allá de los maltratadores y de los factores de riesgo. Madrid: Ministerio de Igualdad.

Bonino, L. (2008). Micromachismos, el poder masculino en la pareja moderna. Recuperado de http://vocesdehombres.files.wordpress.com/2008 /07/micromachismos-el-poder-masculino-en-lapareja-moderna.pdf.

Bosch Fiol, E., Ferrer Pérez, V.A., García Buades, M.E., Ramis Palmer, M.C., Mas Tous, M.C,, Navarro Guzmán, C., et ál. (2007). Del mito del amor romántico a la violencia contra las mujeres en la pareja. Madrid: Ministerio de Igualdad. Secretaría General de Políticas de Igualdad. Instituto de la Mujer.

Delegación del Gobierno para la Violencia de Género. (2013). La evolución de la adolescencia española sobre la igualdad y la prevención de la violencia de género. Madrid: Ministerio de Sanidad, Servicios Sociales e Igualdad. Recuperado de https://www.msssi.gob.es/ssi/violenciaGenero/p ublicaciones/estudiosinvestigaciones/PDFS/Evol _Adolescencia_Prevencion_VG.pdf.

Delegación del Gobierno para la Violencia de Género. (2012). La juventud universitaria ante la igualdad y la violencia de género. Madrid: Ministerio de Sanidad, Servicios Sociales e
Igualdad.

Recuperado

de: https://www.msssi.gob.es/ssi/violenciaGenero/p ublicaciones/estudiosinvestigaciones/PDFS/Juve nt_Universit_Igualdad_VG.pdf.

Federación de Planificación Familiar Estatal. (2012). Factores que influyen en el embarazo de adolescentes y jóvenes: aprendiendo de las experiencias de las jóvenes. Madrid: FPPE.

Hernández, F., Vidiella, J., Herraiz, F., \& Sancho, J.M. (2007). El papel de la violencia en el aprendizaje de las masculinidades. Revista de Educación, 342, 103-125. Recuperado de http://www.revistaeducacion.mec.es/re342/re342 _06.pdf

Kaufman, M. (1999). Los hombres, el feminismo y las experiencias contradictorias del poder entre los hombres. Recuperado de http://www.michaelkaufman.com/wpcontent/uploads/2008/12/los-hombres-elfeminismo-y-las-experiences-contradictoriasdel-poder-entre-los-hombres.pdf.

Moreno Mínguez, A., \& Rodríguez San Julián, E. (2013). Informe de la Juventud en España 2012. Madrid: Ministerio de Sanidad Servicios Sociales e Igualdad. Recuperado de http://www.injuve.es/sites/default/files/2013/26/p ublicaciones/IJE2012_0.pdf.

Muñiz Tinajero, C.O.T. (2012). Satisfacción en la pareja y su relación con sensibilidad a la perspectiva de género, micromachismos, mitos sobre el amor y actitudes sobre el amor (Trabajo Fin de Máster no publicado). Facultad de Psicología. Universidad Complutense de Madrid. Madrid: Publicaciones UCM

Ramírez García, B.O. (2013). El micromachismo, «La violencia invisible». Sapere Aude Atrévete a Pensar, 3(71), 33-38.

Romero von Buchwald, M. (2012). Embarazo adolescente: identidades masculinas y ejercicio de la paternidad (Tesis de Grado no publicada).Quito: Universidad San Francisco de Quito.

Velasco Arias, S. (2009). Recomendaciones para la práctica clínica con enfoque de género. Madrid: Observatorio de Salud de la Mujer. Madrid: Dirección General de la Agencia de Calidad del Sistema Nacional de Salud. Ministerio de Sanidad y Política Social.

Yago Simón, T. (2011). Jóvenes, anticoncepción y género: perspectiva de género en la práctica clínica. Zaragoza: Seminario Interdisciplinar de Estudios de la Mujer de la Universidad de Zaragoza. 


\section{AGRADECIMIENTOS}

- Juan Madrid Gutiérrez. Director del Centro Madrid Salud Joven del Ayuntamiento de Madrid. Facilitador en nuestro estudio.

- Consuelo Soto De Lucía. Matrona y Tutora de la Unidad Docente de Enfermería de Obstetricia y Ginecología del Hospital General Universitario Gregorio Marañón de Madrid.

- Unidad de Investigación para la enfermería, que ayuda a los Residentes de Enfermería (EIR) del Hospital General Universitario Gregorio Marañón de Madrid.

- Instituto de Educación Secundaria Santa Teresa del distrito centro de Madrid, por permitirnos utilizar una de sus aulas para la realización de los grupos de discusión.

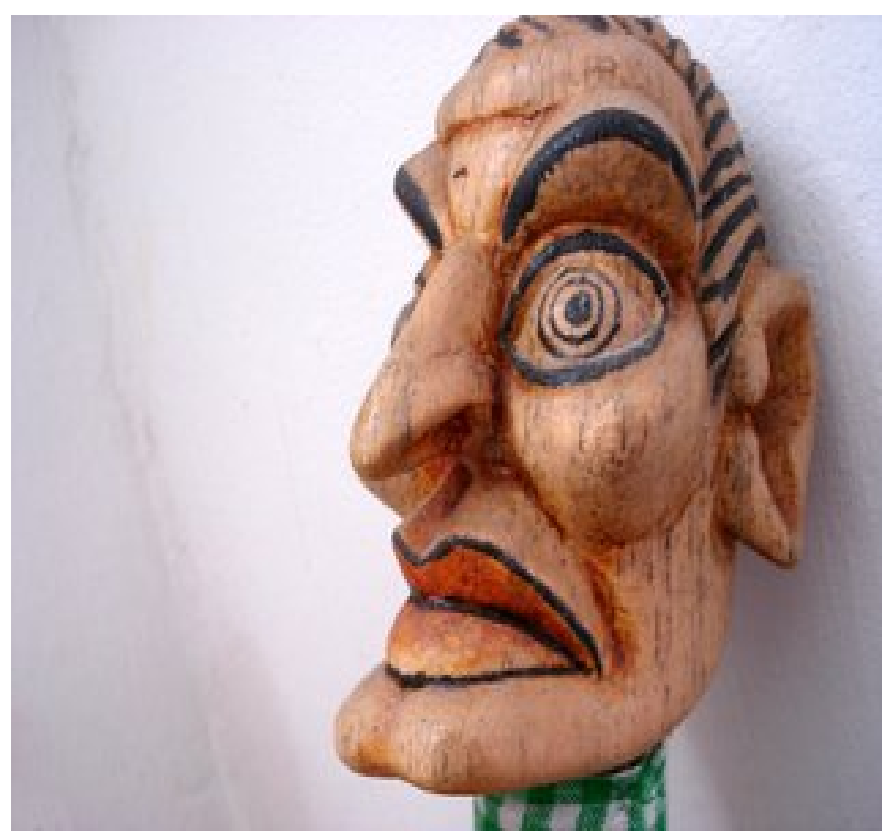

Fuente: https://es.freeimages.com/search/cultura 